\title{
Bidirectional Activity-Dependent Plasticity at Corticostriatal Synapses
}

\author{
Elodie Fino, ${ }^{1}$ Jacques Glowinski, ${ }^{2}$ and Laurent Venance ${ }^{1}$ \\ ${ }^{1}$ Dynamique et Physiopathologie des Réseaux Neuronaux, Institut National de la Santé et de la Recherche Médicale (INSERM) U-667, and 2Neurobiologie \\ Pharmacologique, INSERM U-114, Collège de France, 75231 Paris Cedex 05, France
}

Corticostriatal projections originate from the entire cerebral cortex and provide the major source of glutamatergic inputs to the basal ganglia. Despite the importance of corticostriatal connections in sensorimotor learning and cognitive functions, plasticity forms at these synapses remain strongly debated. Using a corticostriatal slice preserving the connections between the somatosensory cortex and the target striatal cells, we report the induction of both non-Hebbian and Hebbian forms of long-term potentiation (LTP) and long-term depression (LTD) on striatal output neurons (SONs). LTP and LTD can be induced selectively by different stimulation patterns (highfrequency trains vs low-frequency pulses) and were evoked with similar efficiency in non-Hebbian and Hebbian modes. Combination of LTP-LTD and LTD-LTP sequences revealed that bidirectional plasticity occurs at the same SONs and provides efficient homeostatic mechanisms leading to a resetting of corticostriatal synapses avoiding synaptic saturation. The effect of temporal relationship between cortical stimulation and SON activity was assessed using spike-timing-dependent plasticity (STDP) protocols. An LTP was observed when an action potential was triggered in the striatal neuron before the cortical stimulus, and, conversely, an LTD was induced when the striatal neuron discharge was triggered after the cortical stimulation. Such STDP was reversed when compared with those described so far in other mammalian brain structures. This mechanism may be essential for the role of the striatum in learning of motor sequences in which sensory and motor events are associated in a precise time sequence.

Key words: basal ganglia; striatum; cortex; LTP; LTD; homeostasis; spike-timing-dependent plasticity

\section{Introduction}

The cerebral cortex and the basal ganglia constitute tightly interconnected structures involved in sensorimotor learning and cognitive functions (Graybiel, 1995). The striatum, the main input nucleus of basal ganglia, receives massive convergent glutamatergic inputs from the whole cortex. In turn, the striatum exerts a disinhibitory influence on thalamic nuclei by inhibiting the GABAergic neurons of the substantia nigra pars reticulata and of the internal segment of the globus pallidus (the two basal ganglia output nuclei). GABAergic striatal output neurons (SONs), which represent the main neuronal striatal population, act as detectors and integrators of distributed patterns of cortical activity (Graybiel et al., 1994; Wilson, 1995). SONs are silent at rest and need strong and highly time-correlated cortical inputs to discharge. This allows the striatum to detect relevant contextual information from the environment. Because SON discharges finally generate an activation of thalamocortical circuits, the identification of mechanisms regulating transmission at corticostria-

\footnotetext{
Received May 25, 2005; accepted 0ct. 24, 2005.

This work was supported by the Institut National de la Santé et de la Recherche Médicale, Action concertée incitative Jeune Chercheur from the Ministère de la Recherche, Fondation de France Grant 20020111943, and the Collège de France. We thank Dr. Nicolas Maurice and Anne-Marie Godeheu for in vivo experiments and Prof. JeanMichel Deniau and Dr. Dominique Debanne for helpful comments and careful reading of this manuscript.

Correspondence should be addressed to Laurent Venance, Dynamique et Physiopathologie des Réseaux Neuronaux, Institut National de la Santé et de la Recherche Médicale U-667, Collège de France, 75231 Paris Cedex 05, France. E-mail: laurent.venance@college-de-france.fr.

D01:10.1523/JNEUROSCI.4476-05.2005

Copyright $\odot 2005$ Society for Neuroscience $\quad$ 0270-6474/05/2511279-09\$15.00/0
}

tal synapses is essential for a better understanding of basal ganglia function.

Activity-dependent changes in synaptic efficacy may contribute to the formation of precisely organized neural circuits and to learning and memory processes (Malenka and Bear, 2004). Although the striatum is regarded as a major site of memory formation for sensorimotor associations, the occurrence of different forms of plasticity at corticostriatal synapses is still under debate (Calabresi et al., 1996; Mahon et al., 2004).

From previous in vivo and in vitro studies, the inductions of long-term potential (LTP) and long-term depression (LTD) in the striatum were reported to be mutually exclusive and dependent on experimental conditions (Calabresi et al., 1996; Mahon et al., 2004). Moreover, LTD was found to be the preferential form of evoked plasticity. However, if LTP and LTD were indeed mutually exclusive, a resetting of transmission efficacy at these synapses would be impossible, a situation hardly consistent with striatal functions in sensorimotor learning. Therefore, it is essential to determine under which conditions of presynaptic and postsynaptic activity the different forms of plasticity occur at corticostriatal synapses.

In previous experiments in vitro, the stimulation of corticostriatal axons was applied in the corpus callosum or directly in the striatum using slices whose section plane did not strictly conform to the geometry of corticostriatal projections. To obtain specific activation of corticostriatal connections, we used horizontal slices preserving connections between pyramidal neurons of the 
somatosensory cortical area and their striatal target cells. In these conditions, a stimulation applied in the cortical layer $\mathrm{V}$ reliably elicited EPSCs in striatal neurons. We report here for the first time in the same experimental conditions the occurrence of both LTP and LTD at the same corticostriatal synapses using nonHebbian and Hebbian stimulation protocols. By applying a combination of either LTP-LTD or LTD-LTP sequences, we show that these opposite forms of plasticity allow an efficient homeostasis of corticostriatal transmission. Finally, spike-timingdependent plasticity (STDP) protocols were performed to determine the effect of temporal relationship between cortical stimulation and SON activity. Surprisingly, we observed reversed STDP at corticostriatal synapses when compared with those described so far in several mammalian brain structures (Dan and Poo, 2004).

\section{Materials and Methods}

In vivo Neurobiotin injection: anatomical analysis of corticostriatal projections. Corticostriatal neurons were extracellularly labeled with Neurobiotin in Sprague Dawley rats weighting 305-320 g (8 weeks old). All experiments were performed in accordance with local Ethical Committee and European Union guidelines (Directive 86/609/EEC). Briefly, anesthesia was induced by pentobarbital injection $(60 \mathrm{mg} / \mathrm{kg}$, i.p.; Ceva Santé Animale, Libourne, France) and maintained by ketamine local injections (15 mg/kg, i.m., repeated as needed; Merial, Lyon, France). Animals were fixed in a conventional stereotaxic head frame (Horsley Clake Apparatus; Unimécanique, Epinay-sur-Seine, France). Body temperature was maintained at $36.5^{\circ} \mathrm{C}$. Unit recordings were performed with glass micropipettes (2.6 $\mu \mathrm{m}$ external diameter; $12 \mathrm{M} \Omega$ impedance) filled with a solution of $0.5 \mathrm{M} \mathrm{NaCl}$ containing 2.5\% Neurobiotin (Vector Laboratories, Burlingame, CA) and positioned in the somatosensory cortex (anteriority from the interaural line, $7.2 \mathrm{~mm}$; laterality from the midline, $6.0 \mathrm{~mm}$; depth, $2.8 \mathrm{~mm}$ from the cortical surface) according to the atlas of Paxinos and Watson (1986). Neurobiotin was ejected by passing positive current pulses of $2.6 \mu \mathrm{A}$ ( $1 \mathrm{~s} \mathrm{on} / 1 \mathrm{~s}$ off) for $20 \mathrm{~min}$. After an overnight survival, animals were deeply anesthetized with pentobarbital $(130 \mathrm{mg} / \mathrm{kg}$, i.p.) and intracardially perfused with Ringer's solution $(300 \mathrm{ml})$, followed by a 0.1 м PBS, pH $7.4(500 \mathrm{ml})$ containing $4 \%$ paraformaldehyde and $0.1 \%$ glutaraldehyde. Brains were removed, and, after a $2 \mathrm{~h}$ postfixation in a $4 \%$ paraformaldehyde PBS, they were stored for $48 \mathrm{~h}$ in $30 \%$ phosphatebuffered sucrose. Horizontal sections $(70 \mu \mathrm{m})$ were cut using a freezing microtome and collected in $0.1 \mathrm{M}$ PBS, pH 7.4. After several rinses in this buffer, sections were incubated overnight in avidin-biotin-horseradish peroxidase complex (Vectastain $\mathrm{ABC}$ kit; Vector Laboratories) and exposed to a solution of diaminobenzidine $(0.05 \%)$ and $\mathrm{H}_{2} \mathrm{O}_{2}(0.03 \%)$ containing cobalt chloride (1\%) for intensification of the reaction. Sections were then mounted onto gelatin-coated slides and counterstained with safranin.

Electrophysiological recordings. Whole-cell recordings of SONs were performed on horizontal brain slices $(330 \mu \mathrm{m})$ from Sprague Dawley rats (postnatal days 15-21). These slices were prepared at the level of the somatosensory cortical area and of the corresponding corticostriatal projection field. Patch-clamp whole-cell recordings were made as described previously (Venance et al., 2004). Briefly, borosilicate glass pipettes of $15-20 \mathrm{M} \Omega$ resistance contained the following $(\mathrm{mm})$ : $105 \mathrm{~K}$-gluconate, 30 $\mathrm{KCl}, 10$ HEPES, 10 phosphocreatine, 4 ATP-Mg, 0.3 GTP-Na, and 0.3 EGTA, adjusted to $\mathrm{pH} 7.35$ with $\mathrm{KOH}$. The composition of the extracellular solution was the following (mM): $125 \mathrm{NaCl}, 2.5 \mathrm{KCl}, 25$ glucose, 25 $\mathrm{NaHCO}_{3}, 1.25 \mathrm{NaH}_{2} \mathrm{PO}_{4}, 2 \mathrm{CaCl}_{2}, 1 \mathrm{MgCl}_{2}$, and $10 \mu \mathrm{M}$ pyruvic acid (bubbled with $95 \% \mathrm{O}_{2}$ and $5 \% \mathrm{CO}_{2}$ ). All whole-cell recordings were performed at $32^{\circ} \mathrm{C}$ using a temperature control system $(\Delta \mathrm{TC} 3$; Bioptechs, Butler, PA), and slices were continuously superfused at 2-3 $\mathrm{ml} / \mathrm{min}$ with the extracellular solution. Individual neurons were identified using infrared-differential interference contrast microscopy with CCD camera (C2400-07; Hamamatsu, Shizouka, Japan). Signals were amplified using an EPC9-2 amplifier (HEKA Elektronik, Lambrecht, Germany). Current-clamp recordings were filtered at $2.5 \mathrm{kHz}$ and sam- pled at $5 \mathrm{kHz}$, and voltage-clamp recordings were filtered at $5 \mathrm{kHz}$ and sampled at $10 \mathrm{kHz}$ using the program Pulse-8.50 (HEKA Elektronik). Off-line analysis was performed using Igor (WaveMetrics, Lake Oswego, OR). Series resistance compensation was set to $75-80 \%$ in whole-cell configuration $(13.7 \pm 0.9 \mathrm{M} \Omega ; n=145)$. Liquid junction potential error was calculated $(-13.6 \mathrm{mV})$ and corrected.

All chemicals were purchased by Sigma (St. Quentin, France) except 6-cyano-7-nitroquinoxaline-2,3-dione (CNQX) and DL-2-amino-5phosphonopentanoic acid (AP-5) (Tocris Cookson, Ellisville, MO).

Biocytin filling and histochemistry. Biocytin (Sigma) at $5 \mathrm{mg} / \mathrm{ml}$ was dissolved into the patch-clamp pipette solution, and cells were filled during at least $20 \mathrm{~min}$ of recording (performed at $32^{\circ} \mathrm{C}$ ). Subsequently, slices were fixed overnight in $2 \%$ paraformaldehyde at $4^{\circ} \mathrm{C}$. Biocytinfilled cells were visualized using the avidin-biotin-horseradish peroxidase reaction (ABC Elite peroxidase kit; Vector Laboratories) according to the instructions of the manufacturer.

Stimulation protocols. Electrical stimulations of the cerebral cortex were performed with a bipolar electrode (Phymep, Paris, France) placed in the layer $\mathrm{V}$ of the somatosensory cortex. Electrical stimulations were monophasic and at constant current (without detectable polarization of the electrode along time). There was no significant difference of current intensities of cortical stimulations between each stimulation protocol group. This indicates that there was no relationship between the stimulation intensity and the induced plasticities. Currents were adjusted to evoke striatal EPSCs ranging from 50 to $200 \mathrm{pA}$ amplitudes. Repetitive control stimuli were applied at a frequency of $0.1 \mathrm{~Hz}$, a frequency for which no short- and long-term changes in EPSCs amplitudes were induced (supplemental Fig. 1, available at www.jneurosci.org as supplemental material).

Long-term plasticities were investigated by applying either highfrequency stimulation (HFS) (train of $1 \mathrm{~s}$ duration at $100 \mathrm{~Hz}$ repeated four times, separated by $10 \mathrm{~s}$ ) or low-frequency stimulation (LFS) (600 stimuli at $1 \mathrm{~Hz}$ ). HFS and LFS protocols were performed in a Hebbian mode [the depolarization of the postsynaptic element from its resting membrane potential (RMP) to $0 \mathrm{mV}$ was coincident with the presynaptic stimulation] or in a non-Hebbian mode (the postsynaptic element being maintained at its RMP during the presynaptic stimulation). It should be noted that non-Hebbian and Hebbian terms referred strictly to the stimulation protocols and the corresponding induced-plasticities and did not designate underlying induction mechanisms.

STDP experiments consisted in time shifting the presynaptic stimulation with a postsynaptic action potential (AP) evoked by application of suprathreshold depolarizing current pulse. Cortical stimulations and evoked APs in SONs were delivered 100 times at $1 \mathrm{~Hz}$. Neurons were recorded for at least $1 \mathrm{~h}$ after stimulation protocol for long-term plasticities (induced by HFS, LFS, and spike-timing protocols) and at least $2 \mathrm{~h}$ after the first stimulation protocol when LTP and LTD were combined. Input resistances and injected currents were monitored all along the experiments. Variations of these parameters $>20 \%$ lead to the rejection of the experiment.

Data analysis. All results were expressed as mean \pm SEM, and statistical significance was assessed using the Student's $t$ test or the nonparametric Mann-Whitney $U$ test when appropriate at the significance level $(p)$ indicated. Neuronal input resistance was calculated from voltage responses obtained after injecting a hyperpolarizing current ( $-10 \mathrm{pA}, 500$ ms duration). EPSC mean amplitudes were the average of 25 evoked EPSCs for long-term plasticity experiments. Synaptic efficacy changes were classified as either LTP or LTD when the normalized EPSC amplitudes (mean EPSC amplitude after stimulation protocol/mean EPSC amplitude before stimulation protocol) were significantly different from the control baseline. Plasticity loci were determined by the mean variance analysis method (Faber and Korn, 1991; Clements and Silver, 2000). Briefly, EPSCs coefficients of variation (CVs) were calculated by the ratio of the SD and the mean EPSC amplitude. The plasticity loci were deduced from the relationship between the normalized $\mathrm{CV}^{-2}\left(\mathrm{CV}^{-2}\right.$ after induction of plasticity/ $\mathrm{CV}^{-2}$ control) and the normalized EPSC amplitudes (EPSC mean amplitude after induction of plasticity/control EPSC mean amplitude). EPSCs amplitude is proportional to $n p q$, with $n$ being the number of releasing sites, $p$ the probability of release, and $q$ the quantum size. It is admitted that $n$ and $p$ describe presynaptic events, and $q$ is a 

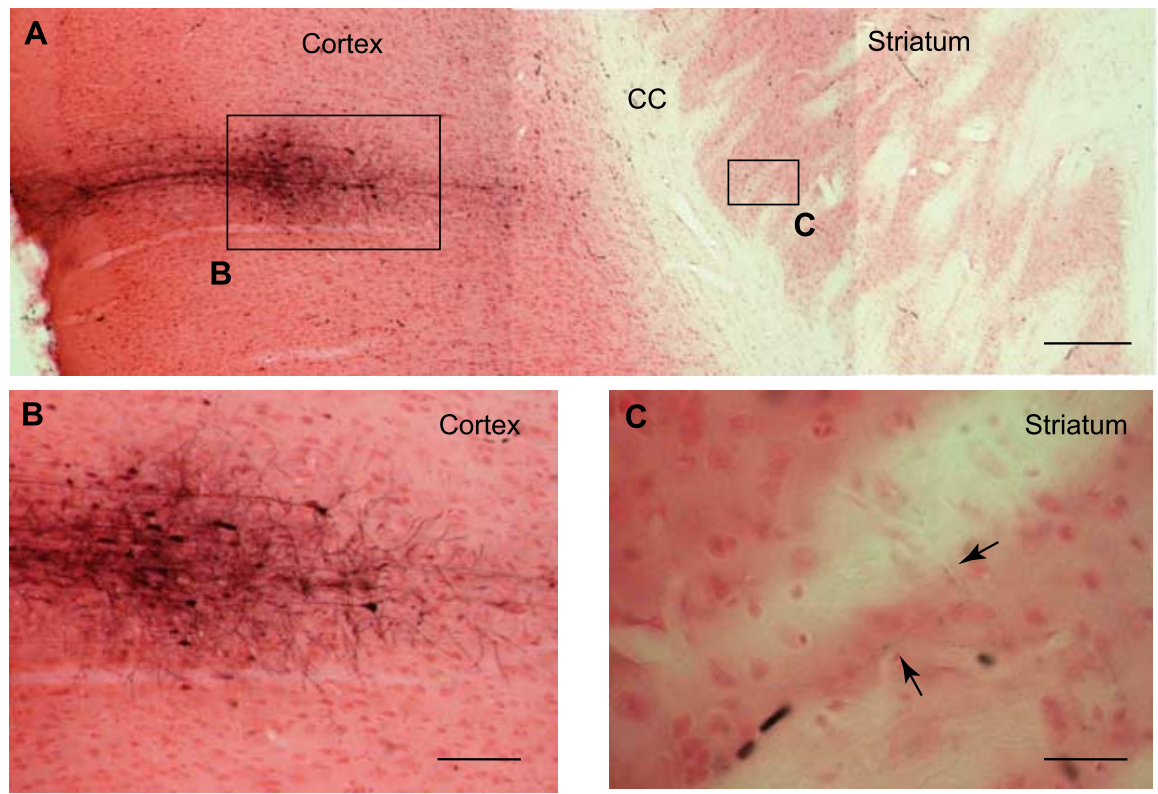

Figure 1. Corticostriatal projections (in vivo Neurobiotin injection). $\boldsymbol{A}$, Microphotograph of a horizontal brain slice obtained from an in vivo juxtacellular Neurobiotin injection (rat at 8 postnatal weeks) performed in the layer IV/V of the somatosensory cortex. Scale bar, $300 \mu \mathrm{m}$. B, Microphotograph showing at a higher magnification the cortical injection site of Neurobiotin. Note, on the right side, the stained pyramidal cells projecting toward the striatum. Scale bar, $100 \mu \mathrm{m}$. C, Stained axons of injected pyramidal cells were visualized in the adjacent striatal area. Scale bar, $50 \mu \mathrm{m}$. CC, Corpus callosum.

postsynaptic indicator. If normalized $\mathrm{CV}^{-2}>$ normalized EPSCs amplitude, both $n$ and $p$ can vary, and if normalized $\mathrm{CV}^{-2}=$ normalized EPSC amplitude, only $n$ can vary. In both cases, changes in EPSC amplitude reflect mainly presynaptic modifications. If normalized $\mathrm{CV}^{-2}<$ normalized EPSC amplitude, changes in EPSC amplitude are related to variations of $n, p$, and $q$, indicating a mixed (presynaptic and postsynaptic) origin of the modifications. Finally, a variation of normalized EPSC amplitude without any variation of normalized $\mathrm{CV}^{-2}$ indicates postsynaptic modifications because only $q$ can vary.

\section{Results}

\section{Reliable corticostriatal transmission}

Corticostriatal projections mainly travel through long and curved pathways that are difficult to keep intact within a brain slice. Anatomical studies have indicated that connections from the somatosensory cortex (layer V) to the striatum are organized in an horizontal plane (Levesque and Parent, 1998). Accordingly, after in vivo Neurobiotin injections in layer $\mathrm{V}$ of this cortical area ( $n=3$ rats), axons of pyramidal cells were visualized as traversing straight across the corpus callosum up to the adjacent striatal area in horizontal slices (Fig. 1). Therefore, stimulations were made in cortical layer $\mathrm{V}$ on horizontal brain slices, and recordings were performed on morphologically and electrophysiologically identified SONs $(n=102)$ (Fig. $2 A-C)$. All experiments were performed at $32^{\circ} \mathrm{C}$ without any pharmacological treatments or ionic modifications to respect the overall effects of local striatal microcircuits involved in corticostriatal transmission. This contrasts with most reported studies in vitro in which GABAergic transmission was blocked and/or ionic concentrations $\left(\mathrm{Mg}^{2+}\right.$ and $\left.\mathrm{Ca}^{2+}\right)$ were modified.

Based on their morphological and electrophysiological properties, SONs were easily distinguished from the striatal interneurons (Kawaguchi et al., 1989; Venance et al., 2004). Morphologically, SONs displayed a medium-sized soma with highly spiny branched dendrites (Fig. $2 B$ ). Electrophysiologically, SONs $(n=$ $102)$ displayed a very hyperpolarized RMP $(-89.0 \pm 0.7 \mathrm{mV})$, a low input resistance $(251 \pm 11 \mathrm{M} \Omega)$, a fast inward rectification, a long depolarizing ramp to spike threshold, a long delay to first spike (384 $\pm 6 \mathrm{~ms})$, and, finally, a medium-range discharge frequency in response to depolarizing current pulses $(12.2 \pm 0.3 \mathrm{~Hz}$ for $+50 \mathrm{pA}$ injected current above AP threshold) (Fig. 2C).

Cortical stimulation evoked PSCs in SONs (Fig. 2D) with a success rate of $90 \%$ $(n=102)$. Once corticostriatal transmission occurred, no failure was observed, and PSC amplitude was stable along time (supplemental Fig. 1, available at www. jneurosci.org as supplemental material). Such reliable cortically evoked postsynaptic events (Fig. 2D) were glutamatergic because they were totally blocked by CNQX $(20 \mu \mathrm{M})$ and AP-5 $(50 \mu \mathrm{M})$ treatments $(n=3)$. Latency times and amplitudes of the cortically evoked EPSCs displayed very narrow Gaussian distributions (Fig. 2E$H)$. When 71 SON corticostriatal transmissions were considered, narrow distributions of latency time (Fig. 2G) and latency $\mathrm{SD}(<1 \mathrm{~ms})$ (Fig. $2 H)$ centered around, respectively, 2.1 and $0.3 \mathrm{~ms}$, denoted a monosynaptic corticostriatal transmission.

\section{Bidirectional long-term plasticity}

The occurrence of both LTP and LTD at corticostriatal synapses was investigated on SONs in slices superfused with a physiological extracellular solution without ionic modification or pharmacological treatment. We investigated first the EPSC amplitude stability during 70 min recordings by applying a $0.1 \mathrm{~Hz}$ stimulation (supplemental Fig. 1, available at www.jneurosci.org as supplemental material). There was no significant variation of EPSC amplitudes along time ( $n=5$ experiments).

At rest, SONs are characterized by a very hyperpolarized RMP and are maintained mostly silent (subthreshold) by intrinsic membrane conductances that shunt uncorrelated cortical inputs (Calabresi et al., 1987; Nisenbaum and Wilson, 1995). Through this filtering process, SONs contribute to the selection of cortical signals (Wickens, 1993; Graybiel et al., 1994; Wilson, 1995). To discharge, SONs require strong and correlated cortical inputs. In the meantime, they can receive other cortical signals when they are already in a suprathreshold state. Therefore, by the use of non-Hebbian (1) and Hebbian (2) stimulation protocols, attempts were made to mimic these two situations of corticostriatal activities: (1) a situation in which depolarization of SONs by cortical afferences remains subthreshold; (2) a situation in which a discharge of convergent corticostriatal neurons leads to a suprathreshold depolarization of SONs.

\section{Long-term potentiation}

HFS (four trains of $1 \mathrm{~s}$ duration at $100 \mathrm{~Hz}$ separated by $10 \mathrm{~s}$ intervals) of the cerebral cortex were applied in both nonHebbian (Fig. 3A) and Hebbian (Fig. 3D) modes. The nonHebbian HFS protocol induced a reliable LTP with a success rate of $80 \%(n=10)$ (see example in Fig. $3 B)$. This non-Hebbian LTP displayed a large increase of EPSC amplitude, which ranged from 87 to $399 \%$ at $45 \min (n=10)$, with a mean value of $221 \pm 33 \%$ 
(Fig. 3C). This value is significantly $(p<$ 0.01 ) different from the baseline recorded in control. Changes in $\mathrm{CV}^{-2}$ ratio related to corresponding normalized mean amplitudes were analyzed to determine the loci of long-term synaptic efficacy modifications. Mean variance analysis indicates whether EPSC potentiation or depression had a presynaptic or a postsynaptic origin (Faber and Korn, 1991; Clements and Silver, 2000). Non-Hebbian LTP appeared to display mainly a presynaptic origin (Fig. $3 C)$.

The Hebbian HFS protocol also induced an LTP of the cortically evoked EPSCs with a success rate of $79 \%(n=14)$ (see example in Fig. 3E). The induced changes of EPSC amplitude ranged from 76 to $598 \%$ at $45 \mathrm{~min}(n=14)$, with a mean value of $249 \pm 46 \%$ (Fig. $3 F$ ). This value was significantly $(p<0.01)$ different from the baseline recorded in control. Mean variance analysis suggested that the Hebbian LTP was mainly underlain by a presynaptic mechanism (Fig. $3 F$ ).

\section{Long-term depression}

Changes in synaptic efficacy induced by cortical LFS (600 stimulations delivered at $1 \mathrm{~Hz}$ ) were also investigated with either non-Hebbian (Fig. 4A) or Hebbian (Fig. $4 D)$ stimulation protocols. Non-Hebbian LFS reliably induced an LTD of the cortically evoked EPSCs with a success rate of $80 \%(n=10)$ (see example in Fig. $4 B$ ). The non-Hebbian LFS protocol induced changes of EPSC amplitude ranged from 11 to $120 \%$ of the control at $45 \mathrm{~min}(n=$ $10)$. The corresponding mean value of EPSC depression at 45 min was $-40 \pm$ $10 \%(n=10)$ and was significantly $(p<$ $0.0001)$ different from the baseline recorded in control (Fig. 4C). Mean variance analysis of the non-Hebbian LTD revealed that synaptic efficacy changes were mainly originated from the postsynaptic element

(Fig. 4C). When Hebbian LFS was used, LTD was also observed with a success rate of $89 \%(n=9)$ (see example in Fig. $4 E$ ). In this case, the changes of EPSC amplitude ranged from 27 to $95 \%$ of the control $(n=9)$. The corresponding mean value of EPSC decrease was $-47 \pm 8 \%$ at $45 \mathrm{~min}$ and was significantly $(p<$ 0.0001 ) different from the baseline recorded in control (Fig. $4 F$ ). Mean variance analysis of Hebbian LTD indicated mainly a postsynaptic origin of such plasticity (Fig. $4 F$ ).

Interestingly, similar high success rates and similar magnitude of EPSC potentiation and depression were observed with nonHebbian and Hebbian HFS and LFS protocols, respectively. As described previously in others systems (Alonso et al., 1990; Urban and Barrionuevo, 1996; Murphy and Glanzman, 1997; Bao et al., 1998) (for review, see Lechner and Byrne, 1998; Sejnowski, 1999; $\mathrm{Bi}$ and Poo, 2001), we report here the existence of bidirectional plasticity induced by non-Hebbian as well as Hebbian protocols at corticostriatal synapses.
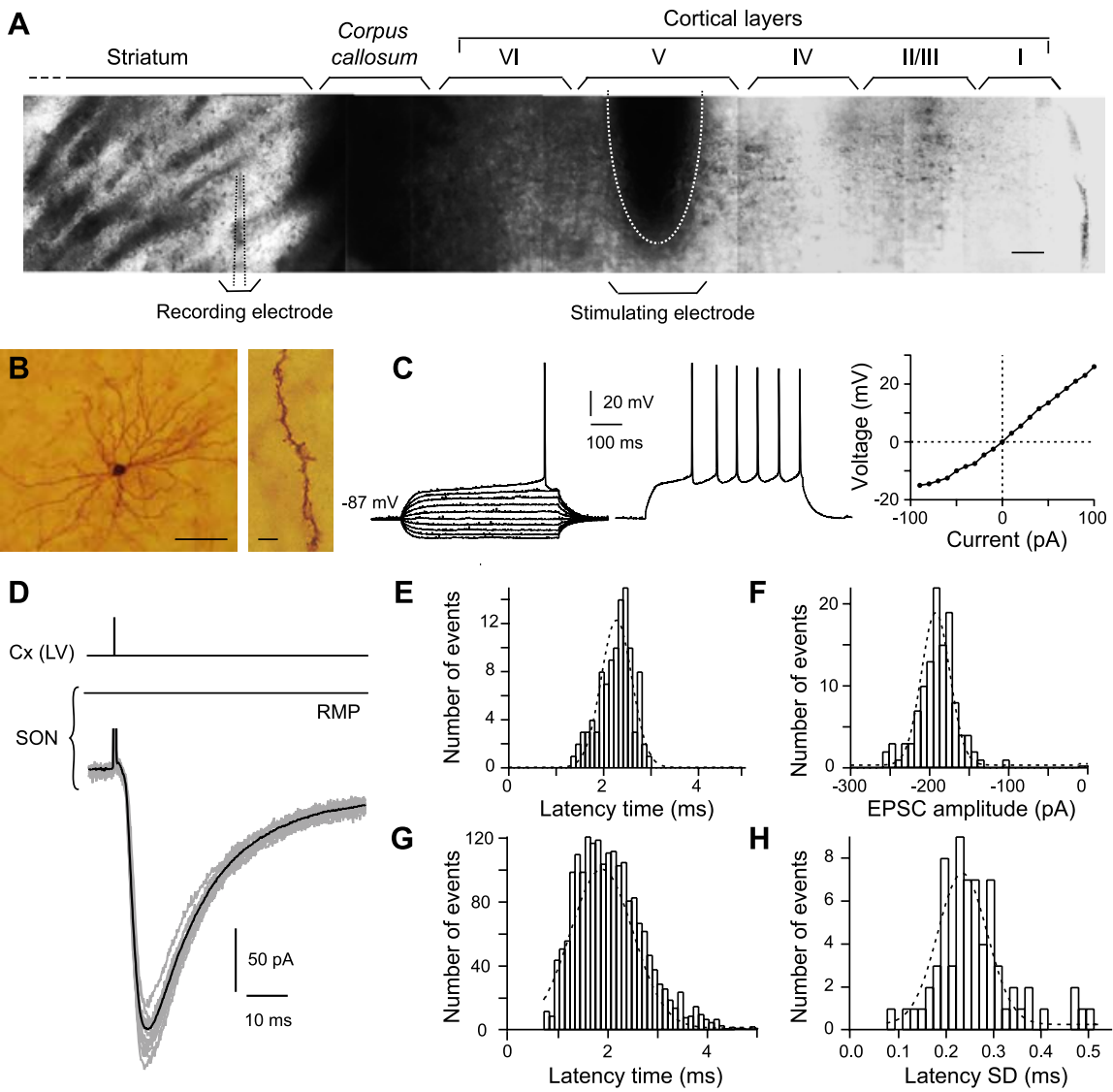

Figure 2. Characteristics of the corticostriatal transmission. $\boldsymbol{A}$, Infrared microphotographs of a horizontal corticostriatal slice 列

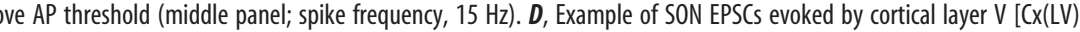
EPSC recorded in a single SON (well fitted by a Gaussian curve). Note the short latency time centered around $2.3 \mathrm{~ms}$. $\boldsymbol{F}$. Amplitude distribution of 103 EPSC amplitudes recorded in the same SON shown in $\boldsymbol{E}$ (distribution well fitted by a Gaussian curve centered around -222 pA). G, Latency distribution of EPSCs recorded in 71 SONs was centered around $2.1 \mathrm{~ms}$ and well fitted by a Gaussian curve. $\boldsymbol{H}$, SD distribution of averaged EPSC latency recorded in 71 SONs was centered around $0.3 \mathrm{~ms}$ and well fitted by a Gaussian curve. Such narrow distributions indicate a monosynaptic corticostriatal transmission.

\section{Corticostriatal homeostatic plasticity}

Because of the high occurrence $(\sim 80 \%)$ of LTP and LTD at corticostriatal synapses, both forms of plasticity could be induced on the same SON, and this should allow to explore interactions between LTP and LTD and therefore the possible existence of homeostatic processes.

Combinations of cortical HFS-LFS and LFS-HFS sequences were thus used to look for the occurrence of both LTP and LTD on the same SON. More precisely, the following sequences of protocols were performed: non-Hebbian HFS followed by nonHebbian LFS, Hebbian HFS followed by Hebbian LFS, nonHebbian LFS followed by non-Hebbian HFS, and Hebbian LFS followed by Hebbian HFS. As shown by representative experiments (Fig. $5 A-D$ ), in both non-Hebbian and Hebbian modes, HFS and LFS protocols induced, respectively, LTP and LTD on the same SON. This demonstrates that bidirectional plasticity takes place at the same corticostriatal synapses. In all cases, long- 

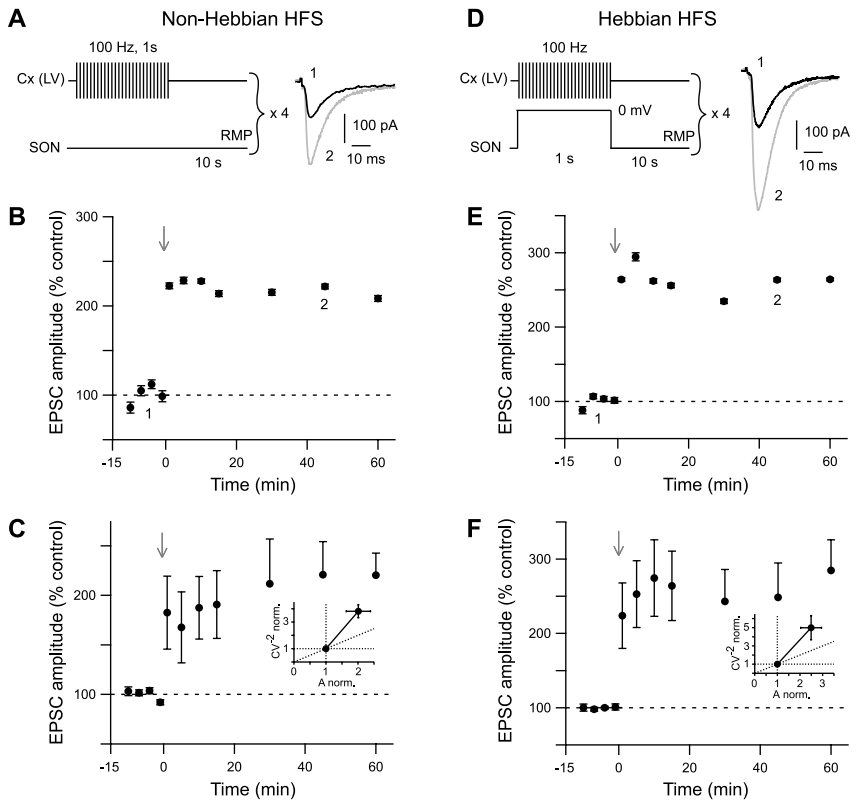

Figure 3. Non-Hebbian and Hebbian HFS protocols induce potent and reliable LTP. A-C, LTP induced by non-Hebbian HFS protocol. $\boldsymbol{A}$, Schematic representation of the non-Hebbian HFS protocol: cortical stimulations (4 trains of $1 \mathrm{~s}$ at $100 \mathrm{~Hz}$, repeated every $10 \mathrm{~s}$ ) were applied while maintaining SONs at their RMP. Raw traces show examples of EPSCs evoked before (1, black trace) and 45 min after (2, gray trace) non-Hebbian HFS protocol. Current traces were taken from the experiment shown in $\boldsymbol{B}$. $\boldsymbol{B}$, Representative experiment of a potent non-Hebbian LTP (222 $\pm 3 \%$ of EPSC amplitude at 45 min; $p<0.0001$ when compared with control baseline) induced by non-Hebbian HFS. C, Averaged data of 10 experiments show potent and sustained LTP of the cortically evoked EPSCs ( $221 \pm 33 \%$ at 45 min; $p<0.01$ when compared with control baseline). $C$, Inset, Mean variance analysis suggests that a presynaptic mechanism underlies the non-Hebbian LTP. Indeed, normalized $\mathrm{CV}^{-2}$ was significantly more than normalized EPSCs amplitude ( $p<0.01$ ) (estimated 45 min after the non-Hebbian LTP induction). $\boldsymbol{D}-\boldsymbol{F}$, LTP induced by Hebbian HFS protocol. $D$, Schematic representation of Hebbian HFS protocol: cortical stimulations ( 4 trains of $1 \mathrm{~s}$ at $100 \mathrm{~Hz}$, repeated every $10 \mathrm{~s}$ ) were applied concomitantly with a $1 \mathrm{~s}$ SON depolarization (from RMP to $0 \mathrm{mV}$ ). $\boldsymbol{E}$, Representative experiment of a potent EPSC potentiation ( $264 \pm 2 \%$ at 45 min; $p<0.0001$ when compared with control baseline) induced by Hebbian HFS. F, Averaged data of 14 experiments show potent and sustained LTP of the cortically evoked EPSCs ( $249 \pm 46 \%$ EPSC potentiation at $45 \mathrm{~min} ; p<0.01$ when compared with control baseline). $\boldsymbol{F}$, Inset, Mean variance analysis of Hebbian LTP indicates a presynaptic origin. Indeed, changes of normalized $\mathrm{CV}^{-2}$ were not significantly different from normalized EPSC amplitude after LTP induction. The gray arrows indicate the stimulation protocols.

term synaptic efficacy changes induced by either HFS or LFS were efficiently counteracted by application of the stimulation protocol eliciting the opposite form of plasticity. Remarkably, no significant difference was observed between EPSC amplitudes in control situations and those recorded on the same cell after either a sequence of LTP followed by LTD $(n=6)$ or LTD followed by an LTP $(n=6)$ (Fig. $5 E)$. Interestingly, such bidirectional plasticity occurred in non-Hebbian as well as Hebbian modes (Fig. $5 E)$. These observations revealed the existence of efficient homeostatic mechanisms at corticostriatal synapses, allowing a resetting of synaptic weights and thereby avoiding synaptic saturation.

\section{Reversed spike-timing-dependent plasticity}

According to several studies performed in structures such as the hippocampus and the cerebral cortex, the temporal relationship between activity in presynaptic and postsynaptic elements constitutes a determinant factor for the induction of synaptic plasticity (Debanne et al., 1994, 1998; Magee and Johnston, 1997; Markram et al., 1997; Bi and Poo, 1998; Egger et al., 1999; Feldman, 2000; Sjostrom et al., 2001; Froemke and Dan, 2002; Tzou-
A
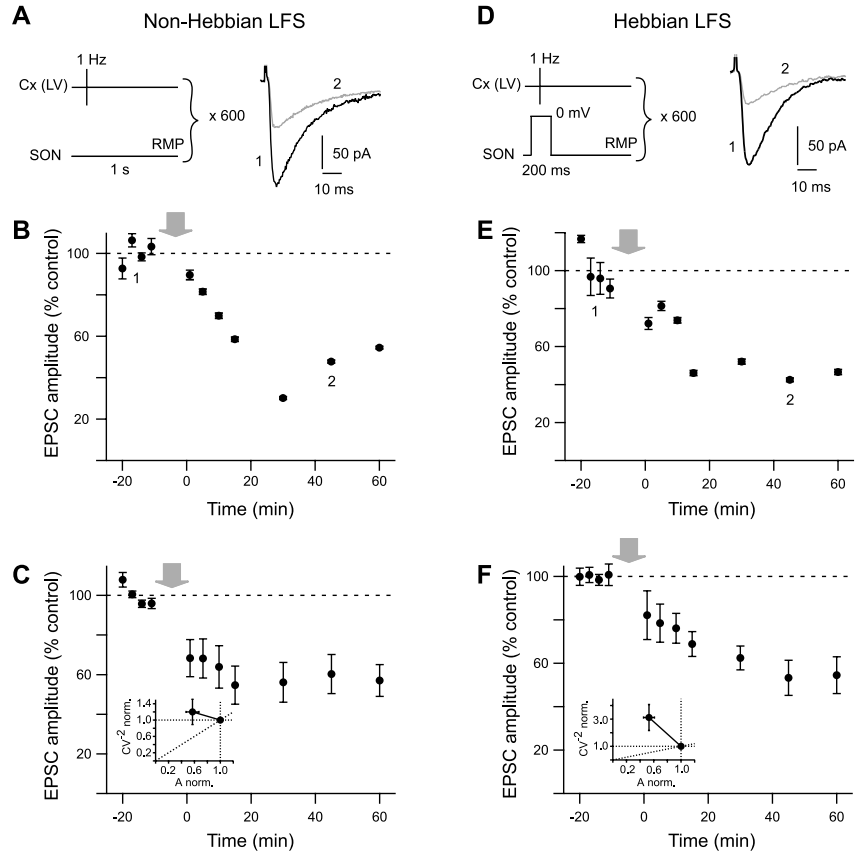

Figure 4. Non-Hebbian and Hebbian LFS protocols induce potent and reliable LTD. $\boldsymbol{A}-\boldsymbol{C}$, LTD induced by non-Hebbian LFS. $\boldsymbol{A}$, Schematic representation of the non-Hebbian LFS protocol: cortical stimulations ( 600 stimulations at $1 \mathrm{~Hz}$ ) were applied while maintaining SONs at their RMP. Raw traces show examples of EPSCs evoked before (1, black trace) and 45 min after (2, gray trace) LFS protocol. $\boldsymbol{B}$, Representative experiment of an effective EPSC depression ( $-47 \pm$ $1 \%$ at 45 min; $p<0.0001$ when compared with control baseline) induced by non-Hebbian LFS. C, Averaged data of 10 experiments show potent and sustained non-Hebbian LTD ( $-40 \pm 10 \%$ EPSC depression at $45 \mathrm{~min} ; p<0.0001$ when compared with control baseline). $\boldsymbol{C}$, Inset, Mean variance analysis revealed that synaptic efficacy changes are mainly originated from the postsynaptic element. Indeed, variation of EPSCs normalized amplitudes occurred without any significant variations of normalized CV ${ }^{-2}$. D-F, Hebbian LFS induced LTD. D, Schematic representation of Hebbian LFS protocol: cortical stimulations ( 600 stimulations at $1 \mathrm{~Hz}$ ) were applied concomitantly to a $200 \mathrm{~ms}$ depolarization of the SON (from RMP to $0 \mathrm{mV}$ ). $\boldsymbol{E}$, Representative experiment of an effective LTD induced by Hebbian LFS $(-43 \pm 1 \%$ EPSC depression at $45 \mathrm{~min}$; $p<0.0001$ when compared with control baseline). $\boldsymbol{F}$, Averaged data of nine experiments show potent and sustained LTD of the cortically evoked EPSCs ( $-47 \pm 8 \%$ EPSC depression at 45 $\min ; p<0.0001$ when compared with control baseline). $\boldsymbol{F}$, Inset, Mean variance analysis indicates mainly a postsynaptic origin of the Hebbian LFS-induced LTD. The gray arrows indicate the stimulation protocols.

nopoulos et al., 2004) (for review, see Dan and Poo, 2004). Therefore, we examined the influence of the temporal relationship between the discharges of SONs and the activation of their cortical afferences on the induction of long-term plasticity. For this purpose, an AP was evoked in the recorded SON by applying a suprathreshold depolarizing current pulse either before or after the stimulation of cortical afferences. This pairing protocol was repeated 100 times at $1 \mathrm{~Hz}$ frequency. An LTP was mainly observed when an AP was triggered in the target SON before the cortical stimulus (Fig. $6 A$, post-pre protocol, $B, E$ ). LTP induction occurred with a success rate of $53 \%(n=15)$ for time intervals from -7 to $-40 \mathrm{~ms}$ (minus sign indicates that postsynaptic AP precedes the presynaptic cortical stimulation). The potentiation of EPSC amplitude estimated at $45 \mathrm{~min}$ after the application of this spike-timing "post-pre" protocol ranged from 87 to $253 \%$, with a mean value of $141 \pm 17 \%(n=15)$. This value was significantly different from the baseline recorded in control $(p<0.05)$ (see averaged baseline data of the post-pre experiments in Fig. $6 E)(n=15)$. No synaptic efficacy changes were observed when the time interval was higher than $-30 \mathrm{~ms}$. Conversely, an LTD was induced (Fig. $6 D, E$ ) when the $\mathrm{SON}$ discharge was triggered 
A

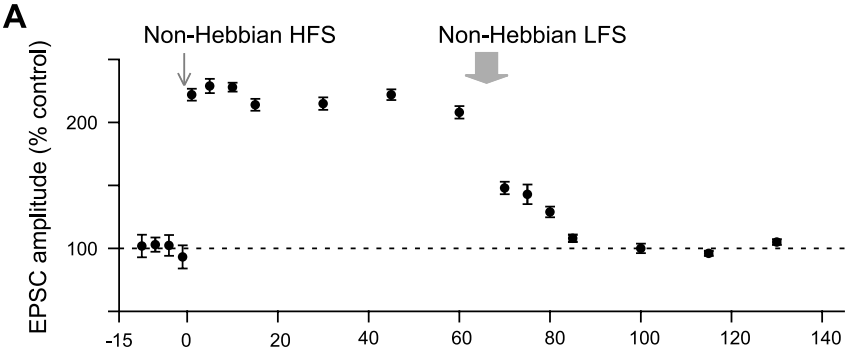

B

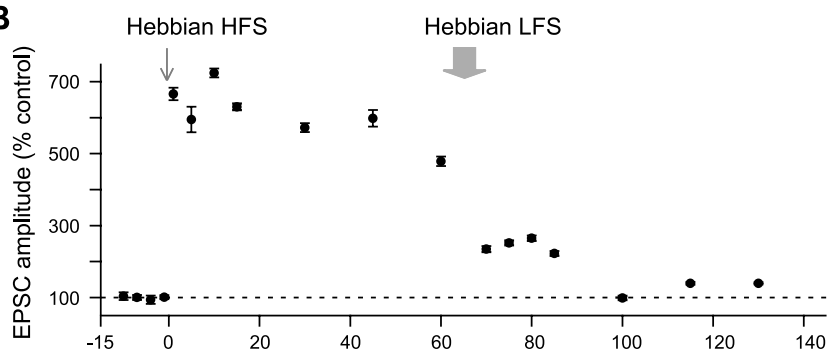

C

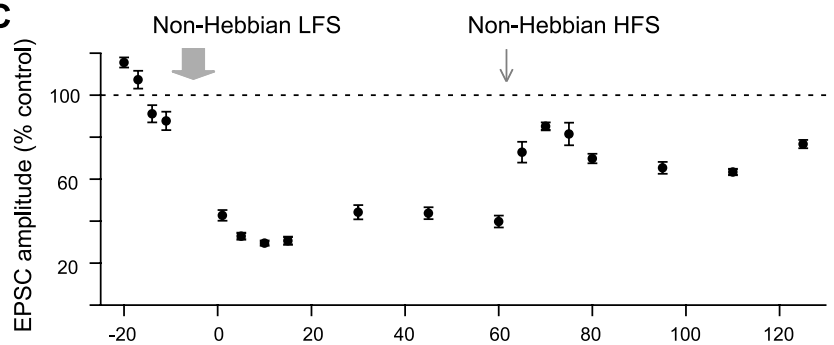

D
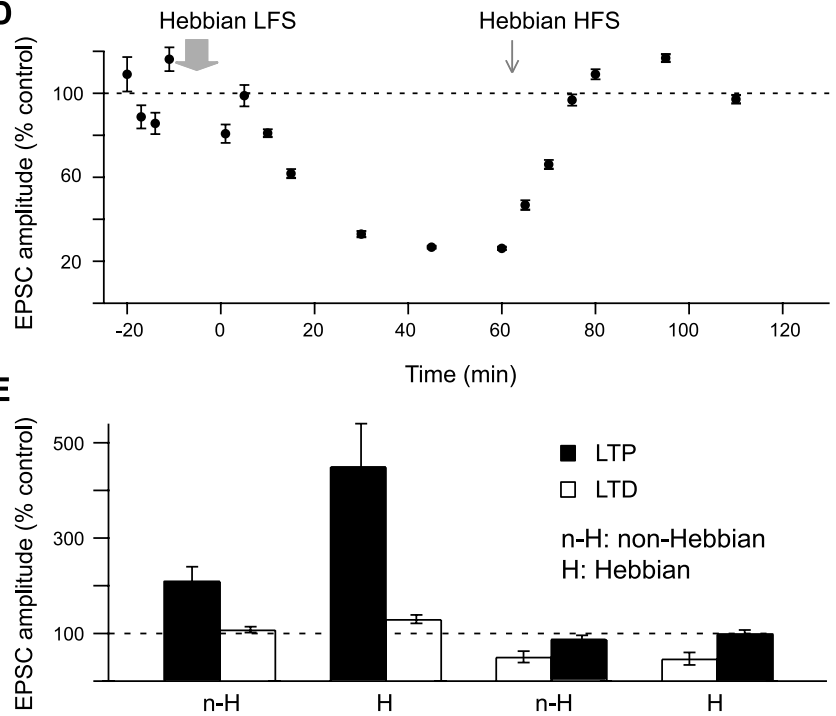

Figure 5. Homeostatic plasticity of corticostriatal transmission. $\boldsymbol{A}$, Representative experiment of a combination of a non-Hebbian HFS followed by a non-Hebbian LFS. The non-Hebbian HFS induced a potentiation of the EPSC amplitude of $222 \pm 3 \%$ (estimated at $45 \mathrm{~min}$ ), and the subsequent non-Hebbian LFS induced a depression of $-126 \%$. The EPSC amplitudes measured at 130 min returned to $96 \pm 1 \%$ of the control. These values were not significantly different from those recorded in control. $\boldsymbol{B}$, Representative experiment of a combination of a Hebbian HFS followed by a Hebbian LFS. Hebbian HFS induced an LTP with a potentiation of the EPSC amplitude of $598 \pm 15 \%$ (estimated at $45 \mathrm{~min}$ ). The subsequent Hebbian LFS induced a depression of $-458 \%$, and EPSC amplitudes measured at 130 min returned to $140 \pm 2 \%$ of the control. $C$, Representative experiment of a non-Hebbian LFS followed by a non-Hebbian HFS. NonHebbian LFS induced an LTD with a depression of the EPSC amplitude of $-66 \pm 1 \%$ (estimated at $45 \mathrm{~min}$ ). The subsequent Hebbian HFS induced a potentiation of 33\%, with EPSC amplitudes measured at 125 min representing $77 \pm 1 \%$ of the control. $\boldsymbol{D}$, Representative experiment of a Hebbian LFS followed by a Hebbian HFS. Hebbian LFS induced an LTD with a depression of the EPSC amplitude of $-73 \pm 1 \%$ (estimated at $45 \mathrm{~min}$ ), and the subsequent after the cortical stimulation (Fig. $6 C$, pre-post protocol). This LTD occurred with a success rate of $67 \%(n=12)$ for time intervals between 5 and $37 \mathrm{~ms}$. The mean EPSC amplitudes estimated at 45 min after the spike-timing "pre-post" protocol ranged from 24 to $117 \%$. The corresponding mean value of EPSC depression was $-25 \pm 9 \%(n=12)$. This value was significantly different from the baseline recorded in control $(p<0.05)$ (see averaged baseline data of the pre-post experiments in Fig. $6 E)(n=12)$. No synaptic efficacy changes were observed when the time interval between the cortical stimulation and the SON discharge was higher than $+30 \mathrm{~ms}$. Mean variance analysis of the cortically evoked synaptic events indicated that the spike-timingdependent LTP arose mainly from the presynaptic element and the spike-timing-dependent LTD had mainly a postsynaptic origin (Fig. 6E). As illustrated in the representative experiments (Fig. 6B,D), the STDP-induced plasticities displayed a longer delay before being fully established when compared with those induced by HFS and LFS protocols. In STDP protocols, being less drastic than HFS and LFS protocols, long-term synaptic efficacy changes might be induced by more subtle intracellular mechanisms, which need longer delays to be fully established.

LTP was induced in SONs when the SON discharge preceded cortical stimulation, whereas LTD was observed when the SON discharge followed the cortical stimulation (Fig. 6E). Interestingly, such responses indicated that the corticostriatal transmission exhibits specific features of STDP because opposite forms of plasticity were found in the other brain structures studied so far in mammals. Indeed, in these structures, an LTP was observed when the discharge of the postsynaptic element followed the activation of the presynaptic element, whereas an LTD occurred when the postsynaptic stimulation preceded the presynaptic stimulation (Debanne et al., 1994, 1998; Magee and Johnston, 1997; Markram et al., 1997; Bi and Poo, 1998; Egger et al., 1999; Feldman, 2000; Sjostrom et al., 2001; Froemke and Dan, 2002; Tzounopoulos et al., 2004) (for review, see Dan and Poo, 2004).

\section{Discussion}

Studies of corticostriatal plasticities required setting up appropriate conditions to record a genuine corticostriatal transmission. Until now, in vitro corticostriatal transmission has been studied indirectly by stimulating in either the corpus callosum or the striatum itself. In addition to activate corticostriatal fibers, such stimulations also recruit extracortical afferences of striatal neurons (GABAergic, serotoninergic, dopaminergic, cholinergic, and noradrenergic) that could orientate plasticity toward either LTP or LTD. Here, we show that stimulations of the somatosensory cortex triggered reliable monosynaptic glutamatergic EPSCs on SONs. The absence of ionic and pharmacological modifications has allowed us to investigate corticostriatal plasticities in a physiological situation that preserves the functionality of the local intrastriatal microcircuits normally involved in the processing of cortical signals.

Hebbian HFS induced a potentiation of 70\%. The EPSC amplitudes measured at 110 min returned to $97 \pm 1 \%$ of the control. This value was not significantly different from the one recorded in control. $\boldsymbol{E}$, Evaluation of the homeostatic plasticity (average of 3 experiments for each combination). In all cases, the second induced plasticity fully counteracted the first one. The amplitudes of EPSCS measured at the end of the second induced plasticity returned to values not significantly different from those of the control. The gray arrows indicate the stimulation protocols. 
A

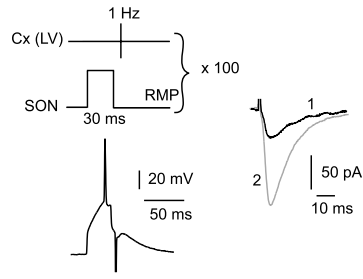

B

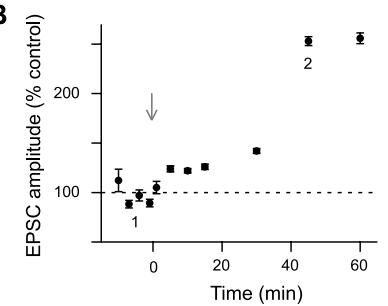

E

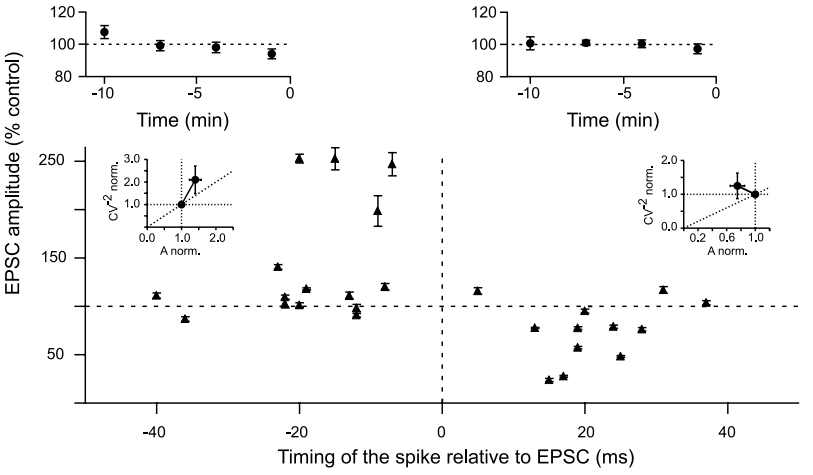

Figure 6. Corticostriatal spike-timing-dependent plasticity. $A$, Post-pre STDP protocol: an AP was evoked in SON (postsynaptic) before the cortical stimulation (100 stimulations at $1 \mathrm{~Hz}$ ). Raw traces illustrate the potent increase of the EPSCs mean amplitudes $45 \mathrm{~min}$ after the STDP post-pre induction (2, gray trace) compared with the control (1, black trace). Traces were taken from the experiment shown in $\boldsymbol{B}$. $\boldsymbol{B}$, Representative experiment of a significant $(p<0.0001)$ LTP induced by the post-pre STDP protocol ( $253 \pm 5 \%$ at 45 min after protocol induction). $C$ Pre-post STDP protocol in which an AP is evoked in SON (postsynaptic) after the cortical stimulation (100 stimulations at $1 \mathrm{~Hz}$ ). Raw traces illustrate the potent decrease of the EPSC mean amplitudes 45 min after the STDP pre-post induction (2, gray trace) compared with the control (1, black trace). Traces were taken from the experiment shown in $\boldsymbol{D}$. $\boldsymbol{D}$, Representative experiment of a significant $(p<0.0001)$ LTD induced by the pre-post STDP protocol $(-52 \pm 1 \%$ at $45 \mathrm{~min}$ after protocol induction). $\boldsymbol{E}$, Spike-timing-dependent changes in synaptic efficacy estimated $45 \mathrm{~min}$ after STDP induction (each recorded SON is indicated by a black triangle). Top graphs show the average of the normalized EPSC amplitudes recorded in control before postpre (top left graph) and pre-post (top right graph) protocols ( $n=15$ and $n=12$, respectively); note the low variability of the control baseline. When an AP was evoked before the cortical stimulation (from -7 to $-40 \mathrm{~ms})$, a significant $(p<0.05)$ LTP $(141 \pm 17 \% ; n=15)$ occurred. No more LTP was observed when the timing of the evoked spike relative to cortical stimulation was more than $-30 \mathrm{~ms}$. When an AP was evoked after the cortical stimulation (from +5 to $+37 \mathrm{~ms})$, a significant $(p<0.05) \operatorname{LTD}(-25 \pm 9 \% ; n=12)$ occurred. No more LTD was observed for timing $>30 \mathrm{~ms}$. $E$, Insets, Normalized $\mathrm{CV}^{-2}$ were plotted as a function of the normalized EPSC amplitude to determine the loci of the STDP. LTP is mainly underlain by presynaptic mechanisms, and LTD has mainly a postsynaptic origin. The gray arrows indicate the stimulation protocols. Cx(LV), Cortical layer V.

\section{LTP, LTD, and homeostatic plasticity at} corticostriatal synapses

As already underlined, long-term plasticity at corticostriatal synapses is still under debate (Calabresi et al., 1996; Mahon et al., 2004). Indeed, pioneering studies in vitro suggested that LTD was the major form of corticostriatal plasticity (Calabresi et al., 1992a; Lovinger et al., 1993; Ronesi and Lovinger, 2005). In addition, LTP was thought to be pathological because it was necessary to remove $\mathrm{Mg}^{2+}$ to observe this response (Calabresi et al., 1992b). The occurrence of LTD or LTP was dependent on experimental conditions: in vitro versus in vivo, stimulation protocols, dopa- minergic tone, localization of the striatal recording site, and magnesium block of NMDA receptors. In studies in vitro, LTD was induced with either HFS (Calabresi et al., 1992a; Lovinger et al., $1993)$ or LFS $(10 \mathrm{~Hz})$ (Ronesi and Lovinger, 2005). In experiments in vivo, LTD has also been observed using HFS (Reynolds and Wickens, 2000), whereas LTP was shown after HFS (Charpier and Deniau, 1997), LFS (5 Hz) (Charpier et al., 1999), or hyperdopaminergy (Reynolds and Wickens, 2000). Moreover, the occurrence of LTD or LTP depended on the striatal region and development because they were observed in distinct striatal area and not at the same period during ontogenesis (Partridge et al., 2000; Smith et al., 2001). However, the occurrence of both plasticities in the same conditions has never been reported. Consequently, this raises also the central question of the existence of homeostatic processes at these synapses.

Here, using a slice preparation preserving corticostriatal connections, we report the occurrence of both LTP and LTD at the same synapses and in the same experimental conditions. The evoked plasticity forms depended exclusively on the type of stimulation protocols applied. LTP was elicited by HFS and LTD by LFS protocols. Here, we report the existence of bidirectional plasticity induced by non-Hebbian as well as Hebbian stimulation protocols at the same synapses. According to Hebb's postulate, if an association of neuronal events induces a Hebbian plasticity, each single event should be neutral. Hebb's postulate has been extended to example studies reporting the coexistence of Hebbian and non-Hebbian plasticities at the same synapses (Alonso et al., 1990; Urban and Barrionuevo, 1996; Murphy and Glanzman, 1997; Bao et al., 1998) (for review, see Lechner and Byrne, 1998; Sejnowski, 1999; Bi and Poo, 2001). At corticostriatal synapses, a non-Hebbian mode mimics a situation of subthreshold cortical inputs. In contrast, a Hebbian stimulation protocol mimics the synchronization of cortical inputs, from the same or different cortical areas, leading thus to a suprathreshold depolarization of SONs. Studies performed in other brain structures report that non-Hebbian protocols either fail (Baranyi and Fehér, 1981; Gustafsson et al., 1987) or induce plasticity with lower magnitude than Hebbian protocols (Alonso et al., 1990; Urban and Barrionuevo, 1996). Therefore, we investigated whether LTP and LTD induced by Hebbian protocols displayed larger synaptic efficacy changes than those induced by non-Hebbian protocols. Surprisingly, in our experiments, LTP and LTD were evoked in the same range of synaptic efficacy changes using both non-Hebbian and Hebbian protocols. This indicates that SONs can develop robust long-term plasticities not only when they are first depolarized by other excitatory inputs but also when they are at a resting state. It should be noted that, because of the highly branched dendritic architecture of the SONs, a space-clamp effect could occur. Nevertheless, Carter and Sabatini (2004) have reported that an absence of stimulation never induced any calcium variation at the SON dendritic level, whereas suprathreshold depolarization triggered a calcium rise in the dendrites. Accordingly, it can be assumed that the evoked somatic suprathreshold depolarization used in Hebbian protocol may backpropagate until corticostriatal dendritic connections. Therefore, the similar synaptic efficacy changes triggered by non-Hebbian and Hebbian modes indicate that the main element to induce long-term plasticity could be the frequency of cortical inputs, whatever the SON membrane potential.

Another major observation of our study is that sequences of LTP-LTD and LTD-LTP revealed the existence of efficient homeostatic processes, allowing the resetting of synaptic efficacy changes and thus avoiding synaptic saturation. This means that 
corticostriatal transmission efficacy can easily return to a naive state, allowing SONs to be fully receptive to subsequent synaptic efficacy modifications. Such an observation is coherent with the role of corticostriatal transmission in sensorimotor learning. Indeed, corticostriatal transmission needs to be highly plastic to take into account environmental variations and to deliver appropriate signals toward basal ganglia output nuclei to produce adapted cognitivomotor responses.

\section{Reversed STDP at corticostriatal synapses}

The temporal relationship between cortical activity and SON discharge was explored using STDP protocols. Our results provide the first evidence that corticostriatal network expresses STDP forms that are reversed to those observed so far in different mammalian brain structures (Magee and Johnston, 1997; Markram et al., 1997; Bi and Poo, 1998; Debanne et al., 1998; Egger et al., 1999; Feldman, 2000; Sjostrom et al., 2001; Froemke and Dan, 2002; Dan and Poo, 2004; Tzounopoulos et al., 2004). An LTP occurred when the SON AP happened before a cortical stimulation, whereas an LTD was observed when an AP was triggered in an SON after a cortical stimulation. To ascertain that such a reversed STDP was not attributable to our experimental conditions, comparative experiments were performed in the cerebral cortex. Corticocortical STDP (with pyramidal cells of layer II/III as presynaptic elements and pyramidal cells of layer $\mathrm{V}$ as postsynaptic elements) displayed the "classical" bidirectional plasticity: LTP when the presynaptic stimulation preceded the postsynaptic stimulation and LTD in the reverse situation (data not shown). As for corticostriatal transmission, reversed STDP have also been described in the cerebellum-like structure of the electric fish (Bell et al., 1997) and in the cartwheel cells of the dorsal cochlear nucleus of mammals (Tzounopoulos et al., 2004), but, in the latter case, this was only a partial reversed STDP (pre-post induced LTD, whereas no plasticity was observed for post-pre protocols).

Mechanisms underlying STDP remain under investigations (Bi and Poo, 1998; Koester and Sakmann, 1998; Goldberg et al., 2002; Froemke et al., 2005) (for review, see Dan and Poo, 2004). Several experimental and theoretical attempts were made to evaluate the impact of different parameters on STDP. In fact, the amplitude and broadness of STDP can be modulated by several factors, such as the dendritic location of the stimulation (Froemke et al., 2005), the shape of the postsynaptic depolarization, the RMP (Saudargiene et al., 2005), and the number of coincidence detectors (density of NMDA receptors) (Karmarkar and Buonomano, 2002). However, when considered separately, none of these factors were shown to be determinant for the observation of a reversed STDP. It is conceivable that several combined factors contribute to the STDP orientation. First, the nature of the neurons (excitatory vs inhibitory) could orientate STDP. For instance, studies reporting reversed STDP (Bell et al., 1997) and partial reversed STDP (Tzounopoulos et al., 2004) involve inhibitory neurons. Second, the architecture of dendritic trees could also play a major role in the shaping of the STDP (Goldberg et al., 2002). Accordingly, SONs exhibit a very hyperpolarized RMP, are GABAergic, and have a highly branched spiny dendritic tree, which receive cortical glutamatergic afferences at a proximal level. The combination of these factors could underlie the reversed STDP observed at the corticostriatal transmission. As shown previously in vivo, corticostriatal synapses exhibit a form of short-term intrinsic memory by which an SON spike discharge increases corticostriatal transmission via a change in membrane conductances (Mahon et al., 2003). The present demonstration of LTP with a post-pre protocol provides an additional mechanism by which inputs paired to a previous suprathreshold synaptic activation can be reinforced. This mechanism may be essential for the role of the striatum in learning of motor sequences in which sensory and motor events are associated in a precise time sequence.

For years, LTD was considered as the unique physiological form of activity-dependent plasticity at corticostriatal synapses. Our results demonstrate in the same conditions the occurrence of both LTP and LTD at these synapses. These findings reconcile the corticostriatal plasticity with the general features of plasticity observed in the CNS. Moreover, the bidirectional plasticity observed in our study underlies the existence of efficient homeostatic corticostriatal plasticity. Finally, the reversed STDP observed at corticostriatal synapses appears to be a specific feature of these synapses.

\section{References}

Alonso A, de Curtis M, Llinas R (1990) Postsynaptic Hebbian and nonHebbian long-term potentiation of synaptic efficacy in the entorhinal cortex in slices and in the isolated adult guinea pig brain. Proc Natl Acad Sci USA 87:9280-9284.

Bao JX, Kandel ER, Hawkins RD (1998) Involvement of presynaptic and postsynaptic mechanisms in a cellular analog of classical conditioning at Aplysia sensory-motor neuron synapses in isolated cell culture. J Neurosci 18:458-466.

Baranyi A, Fehér O (1981) Synaptic facilitation requires paired activation of convergent pathways in the neocortex. Nature 290:413-415.

Bell CC, Han VZ, Sugawara Y, Grant K (1997) Synaptic plasticity in a cerebellum-like structure depends on temporal order. Nature $387: 278-281$.

Bi GQ, Poo MM (1998) Synaptic modifications in cultured hippocampal neurons: dependence on spike timing, synaptic strength, and postsynaptic cell type. J Neurosci 18:10464-10472.

Bi GQ, Poo MM (2001) Synaptic modification by correlated activity: Hebb's postulate revisited. Annu Rev Neurosci 24:139-166.

Calabresi P, Misgeld U, Dodt HU (1987) Intrinsic membrane properties of neostriatal neurons can account for their low level of spontaneous activity. Neuroscience 20:293-303.

Calabresi P, Maj R, Pisani A, Mercuri NB, Bernardi G (1992a) Long-term synaptic depression in the striatum: physiological and pharmacological characterization. J Neurosci 12:4224-4233.

Calabresi P, Pisani A, Mercuri NB, Bernardi G (1992b) Long-term potentiation in the striatum is unmasked by removing the voltage-dependent magnesium block of NMDA receptor channels. Eur J Neurosci 4:929-935.

Calabresi P, Pisani A, Mercuri NB, Bernardi G (1996) The corticostriatal projection: from synaptic plasticity to dysfunctions of the basal ganglia. Trends Neurosci 19:19-24.

Carter AG, Sabatini BL (2004) State-dependent calcium signalling in dendritic spines of striatal medium spiny neurons. Neuron 44:483-493.

Charpier S, Deniau JM (1997) In vivo activity-dependent plasticity at cortico-striatal connections: evidence for physiological long-term potentiation. Proc Natl Acad Sci USA 94:7036-7040.

Charpier S, Mahon S, Deniau JM (1999) In vivo induction of striatal longterm potentiation by low-frequency stimulation of the cerebral cortex. Neuroscience 91:1209-1222.

Clements JD, Silver RA (2000) Unveiling synaptic plasticity: a new graphical and analytical approach. Trends Neurosci 23:105-113.

Dan Y, Poo MM (2004) Spike timing-dependent plasticity of neural circuits. Neuron 44:23-30.

Debanne D, Gahwiler BH, Thompson SM (1994) Asynchronous pre- and postsynaptic activity induces associative long-term depression in area CA1 of the rat hippocampus in vitro. Proc Natl Acad Sci USA 91:1148-1152.

Debanne D, Gahwiler BH, Thompson SM (1998) Long-term synaptic plasticity between pairs of individual CA3 pyramidal cells in rat hippocampal slice cultures. J Physiol (Lond) 507:237-247.

Egger V, Feldmeyer D, Sakmann B (1999) Coincidence detection and 
changes of synaptic efficacy in spiny stellate neurons in rat barrel cortex. Nat Neurosci 2:1098-1105.

Faber DS, Korn H (1991) Applicability of the coefficient of variation method for analyzing synaptic plasticity. Biophys J 60:1288-1294.

Feldman DE (2000) Timing-based LTP and LTD at vertical inputs to layer II/III pyramidal cells in rat barrel cortex. Neuron 27:45-56.

Froemke RC, Dan Y (2002) Spike-timing-dependent synaptic modification induced by natural spike trains. Nature 416:433-438.

Froemke RC, Poo MM, Dan Y (2005) Spike-timing-dependent synaptic plasticity depends on dendritic location. Nature 434:221-225.

Goldberg J, Holthoff K, Yuste R (2002) A problem with Hebb and local spikes. Trends Neurosci 25:433-435.

Graybiel AM (1995) Building action repertoires: memory and learning functions of the basal ganglia. Curr Opin Neurobiol 5:733-741.

Graybiel AM, Aosaki T, Flaherty AW, Kimura M (1994) The basal ganglia and adaptive motor control. Science 265:1826-1831.

Gustafsson B, Wigström H, Abraham WC, Huang YY (1987) Long-term potentiation in the hippocampus using depolarizing current pulses as the conditioning stimulus to single volley synaptic potentials. J Neurosci 7:774-780.

Karmarkar UR, Buonomano DV (2002) A model of spike-timing dependent plasticity: one or two coincidence detectors? J Neurophysiol 88:507-513.

Kawaguchi Y, Wilson CJ, Emson PC (1989) Intracellular recording of identified neostriatal patch and matrix spiny cells in a slice preparation preserving cortical inputs. J Neurophysiol 62:1052-1068.

Koester HJ, Sakmann B (1998) Calcium dynamics in single spines during coincident pre- and postsynaptic activity depend on relative timing of back-propagating action potentials and subthreshold excitatory postsynaptic potentials. Proc Natl Acad Sci USA 95:9596-9601.

Lechner HA, Byrne JH (1998) New perspectives on classical conditioning: a synthesis of Hebbian and non-Hebbian mechanisms. Neuron 20:355-358.

Levesque M, Parent A (1998) Axonal arborization of corticostriatal and corticothalamic fibers arising from prelimbic cortex in the rat. Cereb Cortex 8:602-613.

Lovinger DM, Tyler EC, Merritt A (1993) Short- and long-term synaptic depression in rat neostriatum. J Neurophysiol 70:1937-1949.

Magee JC, Johnston D (1997) A synaptically controlled, associative signal for Hebbian plasticity in hippocampal neurons. Science 275:209-213.

Mahon S, Casassus G, Mulle C, Charpier S (2003) Spike-dependent intrinsic plasticity increases firing probability in rat striatal neurons in vivo. J Physiol (Lond) 550:947-959.

Mahon S, Deniau JM, Charpier S (2004) Corticostriatal plasticity: life after the depression. Trends Neurosci 27:460-467.
Malenka RC, Bear MF (2004) LTP and LTD: an embarrassment of riches. Neuron 44:5-21.

Markram H, Lubke J, Frotscher M, Sakmann B (1997) Regulation of synaptic efficacy by coincidence of postsynaptic APs and EPSPs. Science 275:213-215.

Murphy GG, Glanzman DL (1997) Mediation of classical conditioning in Aplysia californica by long-term potentiation of sensorimotor synapses. Science 278:467-471.

Nisenbaum ES, Wilson CJ (1995) Potassium currents responsible for inward and outward rectification in rat neostriatal spiny projection neurons. J Neurosci 15:4449-4463.

Partridge JG, Tang KC, Lovinger DM (2000) Regional and postnatal heterogeneity of activity-dependent long-term changes in synaptic efficacy in the dorsal striatum. J Neurophysiol 84:1422-1429.

Paxinos G, Watson C (1986) The rat brain in stereotaxic coordinates. Sydney: Academic.

Reynolds JN, Wickens JR (2000) Substantia nigra dopamine regulates synaptic plasticity and membrane potential fluctuations in the rat neostriatum, in vivo. Neuroscience 99:199-203.

Ronesi J, Lovinger DM (2005) Induction of striatal long-term synaptic depression by moderate frequency activation of cortical afferents in rat. J Physiol (Lond) 562:245-256.

Saudargiene A, Porr B, Worgotter F (2005) Local learning rules: predicted influence of dendritic location on synaptic modification in spike-timingdependent plasticity. Biol Cybern 92:128-138.

Sejnowski TJ (1999) The book of Hebb. Neuron 24:773-776.

Sjostrom PJ, Turrigiano GG, Nelson SB (2001) Rate, timing, and cooperativity jointly determine cortical synaptic plasticity. Neuron 32:1149-1164.

Smith R, Musleh W, Akopian G, Buckwalter G, Walsh JP (2001) Regional differences in the expression of corticostriatal synaptic plasticity. Neuroscience 106:95-101.

Tzounopoulos T, Kim Y, Oertel D, Trussell LO (2004) Cell-specific, spike timing-dependent plasticities in the dorsal cochlear nucleus. Nat Neurosci 7:719-725.

Urban N, Barrionuevo G (1996) Induction of Hebbian and non-Hebbian mossy fiber long-term potentiation by distinct patterns of high-frequency stimulation. J Neurosci 16:4293-4299.

Venance L, Glowinski J, Giaume C (2004) Electrical and chemical transmission between striatal GABAergic output neurones in rat brain slices. J Physiol (Lond) 559:215-230.

Wickens JA (1993) Theory of the striatum. Oxford: Pergammon.

Wilson CJ (1995) Models of information processing in the basal ganglia. Cambridge, MA: MIT. 